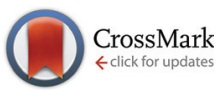

Cite this: Org. Biomol. Chem., 2015, 13,1629

Received 10th October 2014, Accepted 2nd December 2014

DOI: $10.1039 / c 4 o b 02169 j$

www.rsc.org/obc

\section{A short, rigid linker between pyrene and guanidiniocarbonyl-pyrrole induced a new set of spectroscopic responses to the ds-DNA secondary structure $\uparrow$}

\author{
Marijana Radić Stojković, ${ }^{\text {a }}$ Patryciusz Piotrowski, ${ }^{\mathrm{b}}$ Carsten Schmuck* ${ }^{\star \mathrm{b}}$ and \\ Ivo Piantanida*a
}

\begin{abstract}
A novel pyrene-guanidiniocarbonyl-pyrrole dye, characterised by a short, rigid linker between the two chromophores, interacts strongly with ds-DNA but only negligibly with ds-RNA. Under neutral conditions the dye shows strong selectivity toward AT-DNA (with respect to GC-DNA). Binding is accompanied by a specific ICD band at $350 \mathrm{~nm}$ and fluorescence quenching for all DNAs/ RNAs studied. At pH 5 the affinity of the dye is reversed, now favouring GC-DNA over AT-DNA. A strong emission increase for AT-DNA is observed but with quenching for GC-DNA.
\end{abstract}

\section{Introduction}

Versatile applications of small molecules targeting DNA/RNA in biochemical and biomedicinal applications have attracted enormous interest for more than 60 years. ${ }^{1,2}$ The research on DNA/RNA dyes was mostly focused on the impact on the DNA/RNA function or selective/specific DNA/RNA labelling. ${ }^{3,4}$ However, the huge complexity of DNA-coded processes, which do not depend only on the corresponding coding DNA basepair sequence but also include epigenetics, only recently attracted attention. ${ }^{5}$ To address such complex systems, during the last two decades researchers often combined two or more DNA/RNA binding modes in designing novel small molecules used for nucleic acid sensing. Especially specific 3-D bindingmotifs are of interest to achieve such highly selective or specific interactions even with slightly different DNA/RNA structures. ${ }^{2,6}$ For the recognition reporting, fluorescence is the most popular method. ${ }^{7}$ However, upon increasing sensitivity many new techniques or even long established ones are faced with the challenge to circumvent fluorescence disadvantages.

${ }^{a}$ Division of Organic Chemistry and Biochemistry, Ruder Bošković Institute, P. O. Box 180, HR-10002 Zagreb, Croatia. E-mail: Ivo.Piantanida@irb.hr

${ }^{b}$ Institute for Organic Chemistry, University of Duisburg-Essen, Universitässtrasse 7 , 45141 Essen, Germany. E-mail: carsten.schmuck@uni-due.de; Fax: (+) 2011834259 $\dagger$ Electronic supplementary information (ESI) available: Experimental details, synthesis, spectrophotometric data, additional DNA/RNA binding data. See DOI: $10.1039 / \mathrm{c} 4 \mathrm{ob} 02169 \mathrm{j}$
An alternative is circular dichroism (CD) spectropolarimetry, a useful method to study conformational changes in the secondary structure of polynucleotides. ${ }^{8} \mathrm{CD}$ can also be used to study binding interactions as small achiral dyes can eventually acquire an induced CD spectrum (ICD) upon binding to polynucleotides. ${ }^{9}$ Thus, with currently increasing sensitivity, CD spectropolarimetry offers new possibilities of probing DNA/ RNA structures.

In previous work we already studied a series of DNA/RNAbinding compounds in which pyrene, as a fluorophore, was attached to a guanidiniocarbonyl-pyrrole (GCP) cation, an artificial tailor-made anion binding site, which is capable of interacting with the nucleic acid phosphate backbone compounds. ${ }^{10-13}$ These studies revealed several intriguing properties of such compounds: for instance 1 (Scheme 1, 1+ charge, $\mathrm{pH}$ dependent) showed dual spectroscopic recognition between ds-DNA and ds-RNA at pH 5, 2 (2+ charge, pH dependent) transferred the ICD band recognition pattern between ds-DNA and ds-RNA to neutral conditions, 3 and 4 (1+ charge, $\mathrm{pH}$ dependent and additional H-bonding sites in the linkers) finely tuned fluorescence and ICD sensing between various dspolynucleotides based on minor groove recognition. Here we

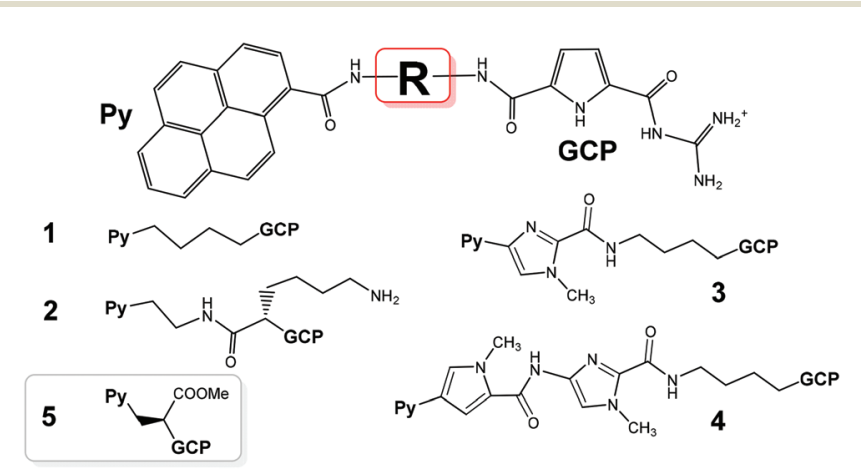

Scheme 1 Previously studied pyrene-guanidiniocarbonyl-pyrrole conjugates 1-4 and the novel derivative studied here (5), which is characterized by the shortest linker studied so far. 
present a novel pyrene analogue 5 characterized by one $\mathrm{pH}$ dependent positive charge (GCP unit) and the shortest linker (with respect to 1-4) of increased rigidity, equipped with a neutral carboxylic-ester side chain. We hope that with a more rigid structure 5 should show increased selectivity in DNA/RNA binding, whereby the carboxylic-ester side chain (neutral to avoid electrostatic repulsion of the negative charges of the nucleic acid) could help to position $\mathbf{5}$ within the DNA/RNA binding site by a combination of steric hindrances and eventual H-bonding interactions. Moreover, 5 has a chiral centre in the main linker chain, which could introduce chiral control of the orientation of the two chromophores.

\section{Results and discussion}

Synthesis of compound $\mathbf{5}$ was performed according to Scheme 2. First a pyrene functionalized amino acid 12 was synthesized, which was then coupled to a Boc-protected guanidiniocarbonylpyrrole derivative 14. Details of the synthesis and characterization of new compounds are given in ESI. $\dagger$

\section{Physico-chemical and spectroscopic properties of aqueous solutions of 5}

Due to the low solubility of $\mathbf{5}$ a stock solution was prepared in DMSO $(0.01 \mathrm{M})$ and solutions for the subsequent studies were prepared by adding small aliquots of this stock solution to
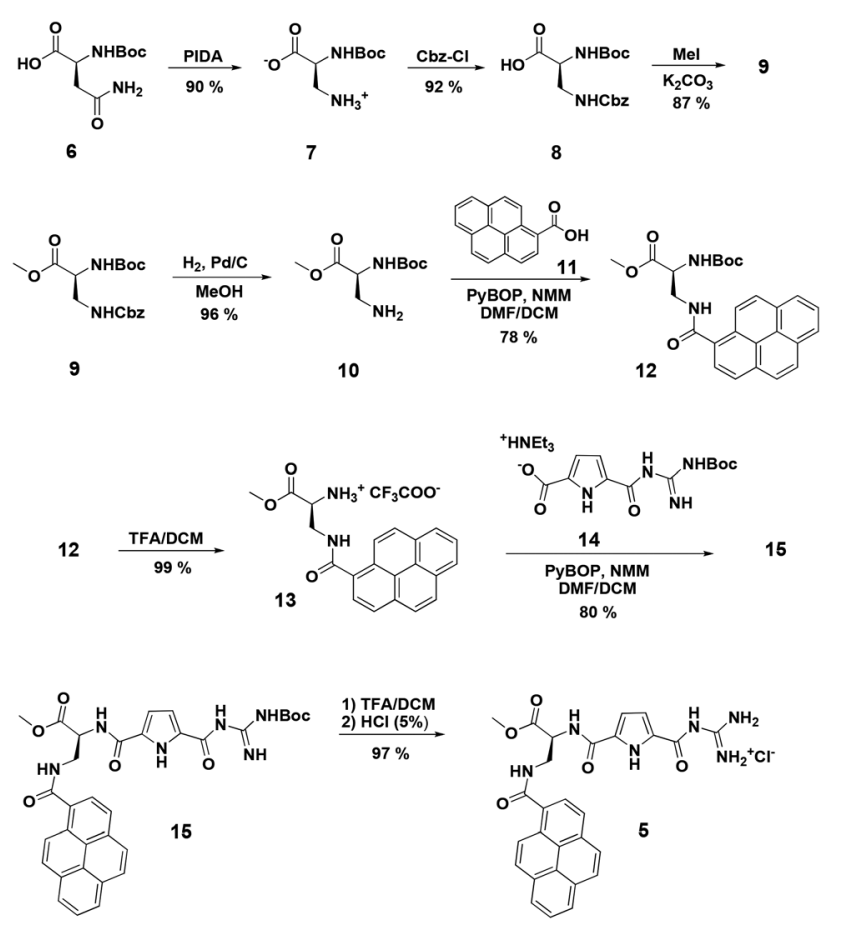

Scheme 2 Synthesis of the pyrene-functionalized amino acid 12 and the new DNA/RNA dye 5 (Boc = butoxycarbonyl, PIDA = (diacetoxyiodo)benzene, $\mathrm{Cbz}=$ carbobenzyloxy, $\mathrm{MeOH}=$ methanol, $\mathrm{PyBOP}=($ benzotriazol-1-yloxy)tri pyrrolidinophosphonium hexafluorophosphate, TFA = trifluoroacetic acid, NMM $=N$-methylmorpholine, $\mathrm{DCM}=$ dichloromethane, $\mathrm{NEt}_{3}=$ triethylamine). buffer solutions (0.05 M Na cacodylate, DMSO content of the final solutions $<0.01 \%$ ). Clear solutions of 5 in the $10^{-5} \mathrm{M}$ range were obtained in this way. At $\mathrm{pH} 7$ compound $\mathbf{5}$ is neutral, while at pH 5 the GCP group is protonated, so that $\mathbf{5}$ bears one positive charge. ${ }^{10,13}$ The concentration dependence of the UV/vis spectrum was linear up to $2 \times 10^{-5} \mathrm{M}$ and the shape of the fluorimetric spectrum showed a well-defined pyrene emission maximum at $\lambda=402 \mathrm{~nm}$ confirming that under these conditions 5 is not aggregated but present as individual molecules in solution. Absorption maxima and the corresponding molar extinction coefficients $(\varepsilon)$ are given in ESI. $\dagger$ Heating of the aqueous solutions of 5 up to $90{ }^{\circ} \mathrm{C}$ did not cause any significant changes in the UV/Vis spectra and reproducibility upon cooling to room temperature verified the chemical stability of the compound.

\section{Interactions with DNA/RNA in aqueous medium}

Because of the significantly different protonation states of $\mathbf{5}$ at different $\mathrm{pH}$ values, binding studies were performed at $\mathrm{pH} 7$ and $\mathrm{pH} 5$. At $\mathrm{pH} 7$ compound 5 is mostly neutral whereas at pH 5 it is mostly protonated ( $\mathrm{p} K_{\mathrm{s}}$ of the GCP unit is around 6). In thermal denaturation experiments at $\mathrm{pH} 5$ and 7, 5 stabilized exclusively AT-DNA ( $\mathrm{pH} 7 / \mathrm{pH} 5, \Delta T_{\mathrm{m}}=3.0 / 9.0^{\circ} \mathrm{C}$, respectively) but not ct-DNA which has a mixed sequence with $42 \%$ GC-basepairs. This is significantly different from the previously studied analogues 1-4. Moreover, 5 did not stabilize ds-RNA. The increased stabilisation effect of 5 at $\mathrm{pH} 5$ can be attributed to increased electrostatic interactions of the now protonated form of $\mathbf{5}$ with the negatively charged backbone of ds-DNA. In line with this interpretation is the observation that compound 2 which is positively charged even at neutral $\mathrm{pH}$ (due to the lysine side chain) shows a significantly stronger binding than 5 at $\mathrm{pH} 7$.

The UV/vis titrations of $\mathbf{5}$ with ds-DNA are characterized by a moderate hypochromic of the absorption band at $\lambda>$ $300 \mathrm{~nm}$, which suggests aromatic stacking interactions. In contrast, the addition of ds-RNA resulted in only negligible changes (ESI Table S2 $\dagger$ ).

Fluorimetric titrations revealed emission changes which depend on the $\mathrm{pH}$, the type of nucleic acid (DNA $v s$. RNA) and the basepair composition of the polynucleotide added. Namely, at pH 7 the emission of $\mathbf{5}$ was quenched by all added ds-DNA/RNA studied (Fig. 1). However, at pH 5 (Fig. 2) upon the addition of AT-DNA the emission considerably increased while the addition of GC-DNA strongly quenched the fluorescence. Again ds-RNA only negligibly influenced the emission of 5 .

Fluorimetric data were processed using the Scatchard equation $^{14}$ to calculate binding parameters (Table 1). The binding affinity of 5 changed considerably between $\mathrm{pH} 7$ and pH 5 (Table 1). Under neutral conditions 5 showed two orders of magnitude higher binding constant for poly $(\mathrm{dA}-\mathrm{dT})_{2}$ in comparison with other ds-polynucleotides. Intriguingly, the absolute change in fluorescence quenching (Fig. 1, I/I $I_{0}$ ) was the smallest upon addition of poly $(\mathrm{dA}-\mathrm{dT})_{2}$ with respect to the other DNA/RNA. This suggests that the fluorophore (pyrene) 


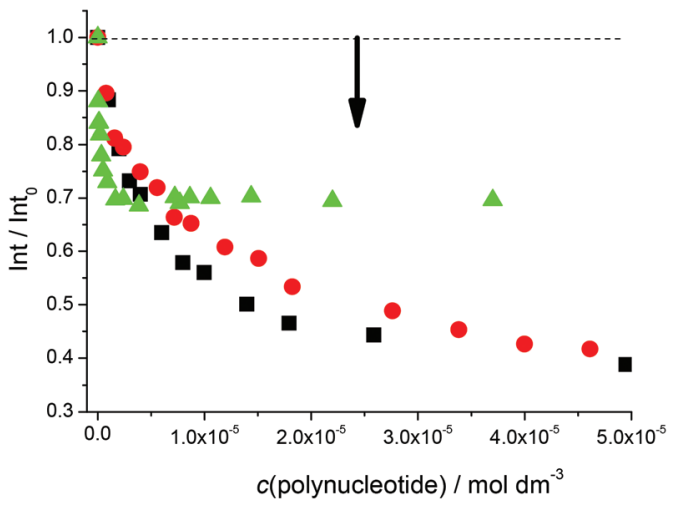

Fig. 1 Normalised fluorescence changes of 5 upon titration at $\mathrm{pH} 7.0$ with poly $(\mathrm{dG}-\mathrm{dC})_{2}\left(\mathbf{\bullet}, c=4.7 \times 10^{-6} \mathrm{M}\right)$, poly A-poly $\mathrm{U}\left(\bullet, c=4.0 \times 10^{-6}\right.$ $\mathrm{M})$, poly $(\mathrm{dA}-\mathrm{dT})_{2}\left(\Lambda, c=1.0 \times 10^{-7} \mathrm{M}\right)$, Na cacodylate buffer, $I=0.05 \mathrm{M}$.

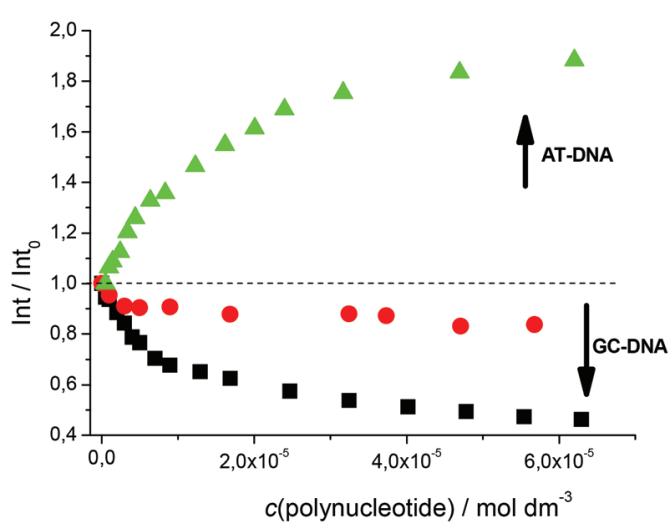

Fig. 2 Normalised fluorescence changes of 5 upon titration at $\mathrm{pH} 5.0$ with poly $(\mathrm{dG}-\mathrm{dC})_{2}\left(\mathbf{\square}, c=1 \times 10^{-7} \mathrm{M}\right)$, poly $\mathrm{A}$-poly $\mathrm{U}\left(\boldsymbol{c}, c=1 \times 10^{-7} \mathrm{M}\right)$, poly $(\mathrm{dA}-\mathrm{dT})_{2}\left(\Delta, c=1.0 \times 10^{-7} \mathrm{M}\right), \mathrm{Na}$ cacodylate buffer, $I=0.05 \mathrm{M}$.

Table 1 Binding constants $\left(\log K_{\mathrm{s}}\right)$, ratios $n=$ [bound compound] $/[$ polynucleotide] of $\mathbf{5}$ and $\mathbf{2}$ with ds-polynucleotides calculated from fluorimetric titrations at $\mathrm{pH} 7$ and $\mathrm{pH} 5$ (buffer sodium cacodylate, $I=0.05 \mathrm{M})^{a}$

\begin{tabular}{lllll}
\hline & & $\begin{array}{l}\text { dAdT-dAdT } \\
\log K_{\mathrm{s}}(n)\end{array}$ & $\begin{array}{l}\text { dGdC-dGdC } \\
\log K_{\mathrm{s}}(n)\end{array}$ & $\begin{array}{l}\text { rA-rU } \\
\log K_{\mathrm{s}}(n)\end{array}$ \\
\hline pH 7 & $\mathbf{5}$ & $7.3(0.3)$ & $5.4(0.9)$ & $5.0(0.9)$ \\
& $2^{c}$ & $6.6(0.2)$ & $5.7(0.2)$ & $b$ \\
pH 5 & $\mathbf{5}$ & $5.3(0.2)$ & $6.4(0.1)$ & $d$ \\
& $2^{c}$ & $6.5(0.3)$ & $6.3(0.2)$ & $5.4(0.2)$
\end{tabular}

${ }^{a}$ Titration data were processed using the Scatchard equation, ${ }^{14}$ accuracy of the obtained $n \pm 10-30 \%$, consequently $\log K_{\mathrm{S}}$ values vary in the same order of magnitude. ${ }^{b}$ Aggregation occurred. ${ }^{c}$ Published results. ${ }^{11,12 d}$ Too small changes for accurate data calculation.

emission response is not proportionally related to the DNA/ RNA binding affinity. Most intriguingly, at pH 5 the selectivity of 5 for a different ds-DNA is reversed (Table 1). For ds-RNA no quantitative binding data could be obtained as negligible fluorimetric changes hampered data processing.

Comparison of the binding parameters with those of the previously studied analogue 2 revealed several distinct differ- ences in $\mathrm{pH}$ dependent affinity as well as in the fluorimetric response. However, fluorimetric titrations showed a very complex pattern of interactions between $\mathbf{5}$ and DNA/RNA, with no straightforward correlations with the structure-activity relationship. Thus for a more detailed structural analysis of the complexes formed, CD spectroscopy was applied. ${ }^{8,9}$ It should be noted that due to its weak intensity the CD spectrum of 5 (ESI $\dagger$ ) could be accurately subtracted from CD spectra of 5/DNA complexes. The CD spectrum of ds-RNA upon titration with 5 did not change significantly (ESI†े). The absence of any ICD bands was attributed to the lack of one dominant and well-structured binding mode of $\mathbf{5}$ with respect to the chiral axis of the RNA double helix.

In contrast to ds-RNA, the addition of 5 to any of the dsDNA studied at either $\mathrm{pH} 5$ or $\mathrm{pH} 7$ resulted in pronounced changes in the CD spectra. Common for all studied DNA was a moderate decrease of the CD band of the DNA itself at $\lambda=$ $245-250 \mathrm{~nm}$, which is characteristic for a distortion of the double stranded helix. For both GC- and AT-DNA isoelliptic points $\lambda<265 \mathrm{~nm}$ supports one dominant type of DNA structure in the complex. However, at $\lambda>265 \mathrm{~nm}$ the observed changes in the CD spectra were strongly influenced by the DNA basepair composition. Spectral changes at $\lambda=$ 265-300 nm arise from several spectroscopically active species (free and bound DNA, ICD effects of bound 5). The individual contributions of each species could not be differentiated from one another.

A more detailed analysis revealed that the CD spectrum of poly dGdC-poly dGdC significantly changed at $\lambda>275 \mathrm{~nm}$ upon addition of 5 (Fig. 3, up) due to the appearance of a strong ICD band at $290-313 \mathrm{~nm}$. As this is the absorption band of the GCP moiety, this ICD band can be attributed to the positioning of the GCP chromophore along one of the DNA grooves. ${ }^{9,10,12}$ Furthermore, the absence of any ICD band $>330 \mathrm{~nm}$ suggests that the pyrene chromophore is not bound in the minor groove but rather outside of the GC-DNA. Most likely steric hindrance between the amino groups of guanine in the minor groove and the sterically demanding linker prevents alignment of the pyrene within the groove. A strongly non-linear dependence of the ICD intensity at $290 \mathrm{~nm}$ pointed to a saturation of the dominant binding site of the GCP moiety at $\left.r_{[5]}\right][$ dGdC-dGdC $]=0.3$.

In contrast to GC-DNA, the addition of 5 to poly dAdT-poly dAdT (Fig. 3, down) resulted in a decrease and a bathochromic shift of the CD band of the DNA at $\lambda=263 \mathrm{~nm}$. Two positive ICD bands appeared at 308 and $350 \mathrm{~nm}$, which agreed nicely with UV/vis maxima of 5 bound to the poly dAdT-poly dAdT complex (ESI, $\uparrow$ Table S2). The ICD band at $350 \mathrm{~nm}$ is specific for the 5/AT-DNA complex as this band is not observed in the 5/GT-DNA complex. This band can be attributed to the uniform positioning of pyrene within the DNA double helix. ${ }^{10,12}$ Furthermore, the positive sign of this ICD band suggests that the pyrene is either positioned along the minor groove $^{9}$ or partially intercalated with its long axis oriented perpendicularly to the long axes of the adjacent DNA basepairs. ${ }^{9}$ For the accurate differentiation between these two binding 

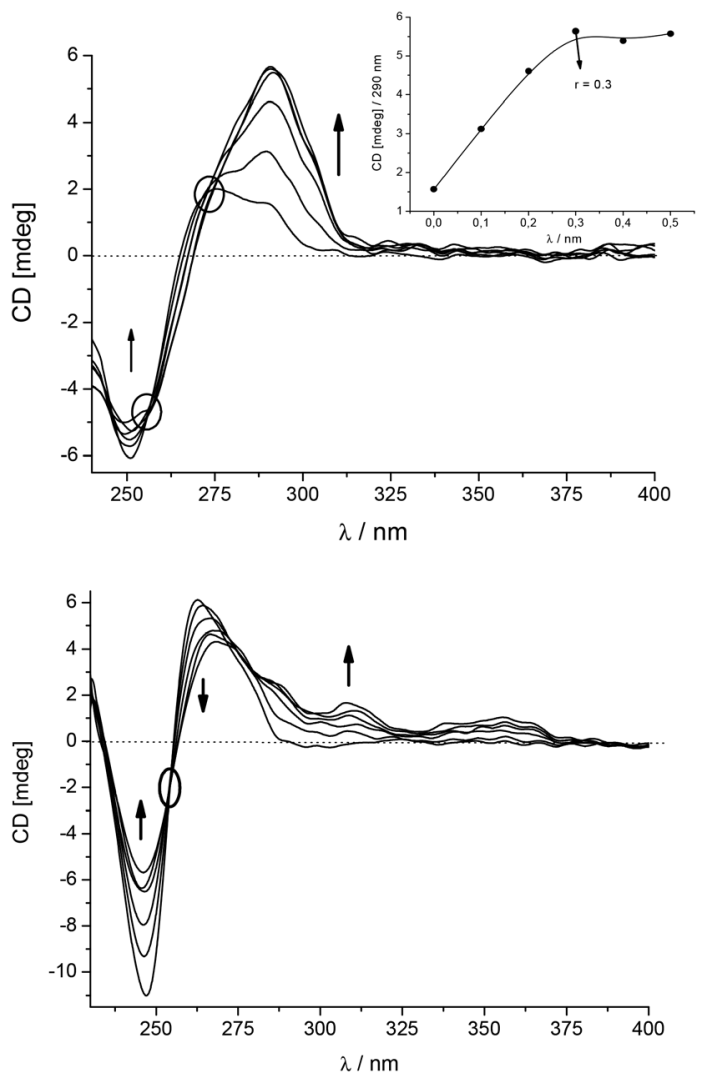

Fig. 3 Changes in CD spectra upon the addition of 5 to poly dGdCpoly dGdC $\left(c=1.0 \times 10^{-5} \mathrm{M}\right)$ (top) and poly dAdT-poly dAdT ( $c=1.0 \times$ $10^{-5} \mathrm{M}$ ) (bottom). Molar ratios $r[5] /[$ polynucleotide] $=0.1,0.2,0.3,0.4$, 0.5 at $\mathrm{pH} 7.0$ ( $\mathrm{N}$ cacodylate buffer, $I=0.05 \mathrm{M}$ ). Inset: dependence of the ICD intensity on the ratio $r$.

modes NMR analysis of 5/oligonucleotide complexes would be necessary, which was however hampered by the insufficient solubility of 5/DNA complexes at concentrations needed for such NMR studies. Therefore, we performed simple molecular docking studies to probe the insertion of $\mathbf{5}$ into AT-DNA as we did previously for the intercalating analogue $\mathbf{1}^{10}$ and the minor groove binder $4 .{ }^{12}$ The obtained structures (ESI $\dagger$ ) show that the rigid linker of $\mathbf{5}$ hampers the efficient insertion of the GCP moiety into the minor groove when the pyrene is intercalated. Thus the insertion of the whole molecule 5 into the minor groove is the most likely binding mode.

The most intriguing observation was that the addition of $\mathbf{5}$ to ct-DNA (58\% AT; $42 \%$ GC) resulted in much smaller changes of the CD spectrum (only weak ICD bands at 290-310 nm, ESI $\dagger$ ) in comparison with synthetic AT- or GC-DNA. Obviously a homogeneous basepair composition favours the specific binding mode, which is characteristic for the observed ICD signature.

\section{Conclusions}

In contrast to the previously studied compound $\mathbf{1}$, which interacted significantly with DNA/RNA only at pH 5 when the GCP moiety is protonated, $\mathbf{2}$ and $\mathbf{5}$ showed significant interactions also at $\mathrm{pH}$ 7. However, there are several distinct differences between 5 and previously studied 1-4. Firstly, only 5 revealed high selectivity toward AT-DNA at $\mathrm{pH} 7$ as confirmed by the two orders of magnitude higher binding constant (Table 1), the exclusive thermal stabilisation of only AT-DNA but of no other DNA/RNA, and the specific positive ICD band at $350 \mathrm{~nm}$, supporting a uniform positioning of the pyrene within the DNA double helix. Secondly, this selectivity of $\mathbf{5}$ was reversed at $\mathrm{pH}$, now favouring GC-DNA over AT-DNA and with no ICD band showing up at $350 \mathrm{~nm}$. Hence, the $\mathrm{pH}$ change induced a different binding mode in which the pyrene is removed from the DNA-double helix. Furthermore, 5 showed only at pH 5 a fluorescence increase exclusively for AT-DNA while GC-DNA yielded emission quenching, for similar reasons as for analogue $2 .{ }^{11}$ Intriguingly, 2 interacted also strongly with ds-RNA, in contrast to the negligibly weak interaction of $\mathbf{5}$. The reason for this is not clear yet.

Most likely these distinct spectrophotometric responses of $\mathbf{5}$, which are significantly different from the previously studied analogues 1-4, can be attributed to its short, rigid linker. This linker only allows two chromophores to adopt very limited orientations upon interaction with the different DNA binding sites. Especially interesting is the strong interaction of neutral 5 with DNA at pH 7, since only very few neutral compounds show biorelevant DNA interactions ( $>90 \%$ DNA dyes are positively charged). ${ }^{2}$

However, a major disadvantage is the low solubility of $\mathbf{5}$, which will be addressed in future research to allow more detailed structural studies of DNA complexes formed (e.g. by NMR studies). Furthermore, future work will also focus on the enantiomer of $\mathbf{5}$, which could show different patterns of DNA/ RNA interactions, similarly to recently reported chiral perylene bisimide derivatives. ${ }^{15}$ Also, biological studies of 5 and comparison with the activity of its close analogues ${ }^{11,12}$ are of the highest interest, to characterize its antiproliferative activity, cellular uptake and distribution. Nonetheless, $\mathbf{5}$ is particularly convenient for further synthetic modifications and the design of second generation derivatives since the carboxy-group allows easy functionalization to structurally even more complicated analogues by simple peptide bond formation, for instance based on the peptide backbone recombination. ${ }^{16}$

\section{Acknowledgements}

Financial supports from the Croatian Science Foundation (grant no. 1477) and FP7-REGPOT-2012-2013-1, grant agreement number 316289 - InnoMol are gratefully acknowledged. I. P. and C. S. are grateful to COST Action CM1005 for the support in networking and mutual cooperation.

\section{Notes and references}

1 R. B. Silverman, The Organic Chemistry of Drug Design and Drug Action, Elsevier Academic Press, New York, 2004. 
2 DNA and RNA Binders, ed. M. Demeunynck, C. Bailly and W. D. Wilson, Wiley-VCH, Weinheim, 2002.

3 E. Trinquet and G. Mathis, Mol. Biosyst., 2006, 2, 381.

4 Y. N. Teo and E. T. Kool, Chem. Rev., 2012, 112, 4221.

5 (a) S. B. Baylin and K. E. Schuebel, Nature, 2007, 448, 548; (b) K. K. Li, C. Luo, D. X. Wang, H. L. Jiang and Y. G. Zheng, Med. Res. Rev., 2012, 32, 815.

6 B. Willis and D. P. Arya, Bioorg. Med. Chem. Lett., 2009, 19, 4974.

7 (a) R. J. Lipshutz, S. P. A. Fodor, T. R. Gingeras and D. J. Lockhart, Nat. Genet., 1999, 21, 20; (b) L. M. Smith, J. Z. Sanders, R. J. Kaiser, P. Hughes, C. Dodd, C. R. Connell, C. Heiner, S. B. H. Kent and L. E. Hood, Nature, 1986, 321, 674; (c) T. Oida, Y. Sako and A. Kusumi, Biophys. J., 1993, 64, 676; (d) A. Hillisch, M. Lorenz and S. Diekmann, Curr. Opin. Struct. Biol., 2001, 11, 201.

8 A. Rodger and B. Nordén, Circular Dichroism and Linear Dichroism, Oxford University Press, New York, 1997, Ch. 2.
9 M. Eriksson and B. Nordén, Methods Enzymol., 2001, 340, 68-98.

10 L. Hernandez-Folgado, C. Schmuck, S. Tomić and I. Piantanida, Bioorg. Med. Chem. Lett., 2008, 18, 2977.

11 L. Hernandez-Folgado, D. Baretić, I. Piantanida, M. Marjanović, M. Kralj, T. Rehm and C. Schmuck, Chem. Eur. J., 2010, 16, 3036.

12 K. Gröger, D. Baretić, I. Piantanida, M. Marjanović, M. Kralj, M. Grabar, S. Tomić and C. Schmuck, Org. Biomol. Chem., 2011, 9, 198.

13 K. Klemm, M. Radić Stojković, G. Horvat, V. Tomišić, I. Piantanida and C. Schmuck, Chem. - Eur. J., 2012, 18, 1352.

14 J. D. McGhee and P. H. von Hippel, J. Mol. Biol., 1976, 103, 679.

15 T. H. Rehm, M. R. Stojković, S. Rehm, M. Škugor, I. Piantanida and F. Wurthner, Chem. Sci., 2012, 3, 3393.

16 M. Dukši, D. Baretić, V. Čaplar and I. Piantanida, Eur. J. Med. Chem., 2010, 45, 2671. 